\title{
Antiferromagnetic order breaks inversion symmetry in metallic double perovskite, $\mathrm{Pb}_{2} \mathrm{NiOsO}_{6}$
}

\author{
Hai L. Feng, ${ }^{1,2}$ Chang-Jong Kang,, 3 Pascal Manuel, ${ }^{5}$ Fabio Orlandi, ${ }^{3}$ Yu Su, $, 6,7$ Jie Chen,, 6 \\ Yoshihiro Tsujimoto, ${ }^{6,7}$ Joke Hadermann, ${ }^{8}$ Gabriel Kotliar, ${ }^{3}$ Kazunari Yamaura, ${ }^{6,7}$ Emma E. \\ McCabe, ${ }^{9,} 1^{*}$ Martha Greenblatt ${ }^{1 *}$
}

${ }^{1}$ Department of Chemistry and Chemical Biology, Rutgers, the State University of New Jersey, 123 Bevier Road, Piscataway, NJ 08854, USA

${ }^{2}$ Beijing National Laboratory for Condensed Matter Physics and Institute of Physics, Chinese Academy of Sciences, Beijing 100190, China

${ }^{3}$ Department of Physics and Astronomy, Rutgers, the State University of New Jersey, 136 Frelinghuysen Road, Piscataway, NJ 08854, USA

${ }^{4}$ Department of Physics, Chungnam National University, Daejeon 34134, South Korea

5ISIS facility, STFC, Rutherford Appleton Laboratory, Chilton, Didcot, Oxfordshire OX11 0QX, UK.

${ }^{6}$ International Center for Materials Nanoarchitectonics (WPI-MANA), National Institute for Materials Science, Tsukuba, Ibaraki 305-0044, Japan

${ }^{7}$ Graduate School of Chemical Sciences and Engineering, Hokkaido University, Sapporo, Hokkaido 060-0810, Japan

${ }^{8}$ EMAT, University of Antwerp, Groenenborgerlaan 171, 2020 Antwerp, Belgium

${ }^{9}$ School of Physical Sciences, University of Kent, Canterbury, Kent CT2 7NH, UK

${ }^{10}$ Department of Physics, Durham University, Lower Mountjoy, South Road, Durham, DH1 3LE, UK

*Martha Greenblatt

greenbla@chem.rutgers.edu

${ }^{*}$ Emma E. McCabe

emma.mccabe@durham.ac.uk 


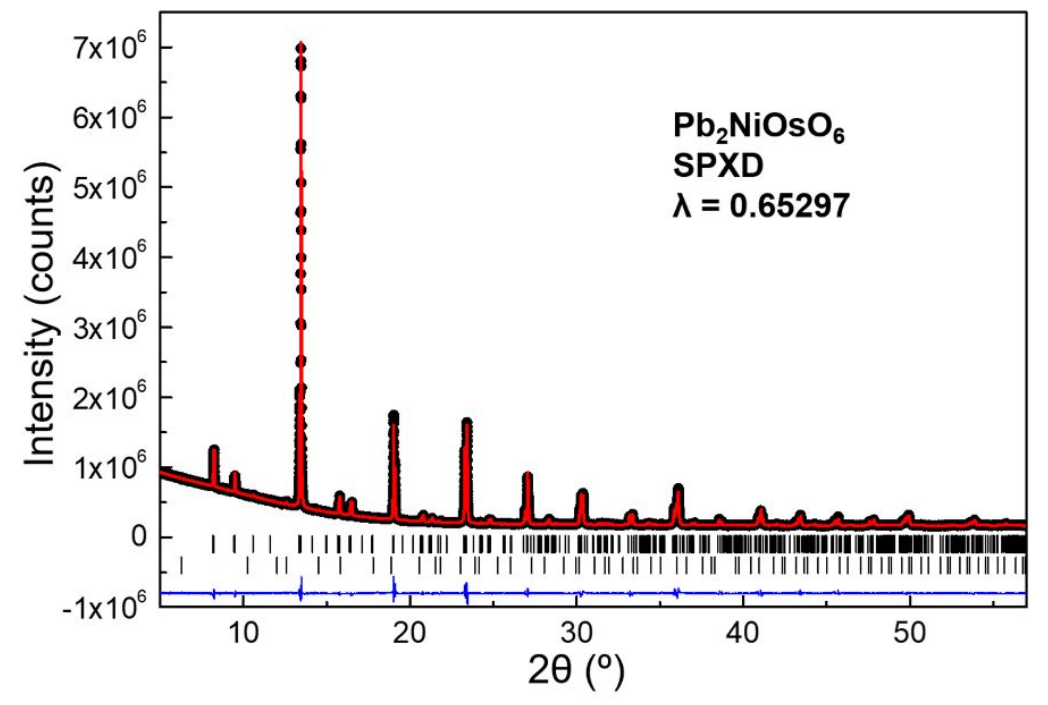

Figure S1 Rietveld refined room temperature SPXD pattern of $\mathrm{Pb}_{2} \mathrm{NiOsO}_{6}$. Black dots and red solid lines show the observed and calculated patterns, respectively. The difference between the observed and calculated patterns is shown as blue lines at the bottom. The vertical bars indicate positions of expected Bragg reflections for the titled compound.

Table S1 Atomic positions and isotropic displacement factors obtained from the SPXD data of $\mathrm{Pb}_{2} \mathrm{NiOsO}_{6}{ }^{*}$

\begin{tabular}{cccccc}
\hline Atom & Site & $x$ & $y$ & $z$ & $B_{\text {iso }}\left(\AA^{2}\right)$ \\
\hline $\mathrm{Pb}$ & $4 e$ & $0.5022(8)$ & $0.5062(4)$ & $0.2503(1)$ & $1.20(1)$ \\
$\mathrm{Ni}$ & $2 c$ & 0.0 & 0.5 & 0.0 & $0.26(1)$ \\
$\mathrm{Os}$ & $2 b$ & 0.5 & 0.0 & 0.0 & $0.26(1)$ \\
$\mathrm{O} 1$ & $4 e$ & $0.254(10)$ & $0.248(7)$ & $1.00(3)$ & $1.0($ fixed $)$ \\
$\mathrm{O} 2$ & $4 e$ & $0.260(10)$ & $0.740(7)$ & $1.00(3)$ & $1.0($ fixed $)$ \\
$\mathrm{O} 3$ & $4 e$ & $0.428(3)$ & $0.927(2)$ & $0.221(2)$ & $1.0($ fixed $)$ \\
\hline
\end{tabular}

*Space group $P 2 / n$ : $a=5.58724(3) \AA, b=5.62988(2) \AA, c=7.89791(3) \AA, \beta=90.001(6)^{\circ} ; R_{w p}=2.16$, $R_{p}=1.16$. 
Table S2 Selected bond distances and bond angles for $\mathrm{Pb}_{2} \mathrm{NiOsO}_{6}$ refined from SPXD and NPD data at different temperatures.

\begin{tabular}{|c|c|c|c|}
\hline Temperature & $298 \mathrm{~K}$ & $98 \mathrm{~K}$ & $1.5 \mathrm{~K}$ \\
\hline Diffraction source & Synchrotron & Neutron & Neutron \\
\hline $\mathrm{Pb}-\mathrm{O}(1)$ & $1 \times 2.82(17) \AA$ & $1 \times 2.470(2) \AA$ & $1 \times 2.464(2)$ \\
\hline $\mathrm{Pb}-\mathrm{O}(1)$ & $1 \times 2.79(18) \AA$ & $1 \times 2.945(3) \AA$ & $1 \times 2.960(2)$ \\
\hline $\mathrm{Pb}-\mathrm{O}(1)$ & $1 \times 2.81(17) \AA$ & $1 \times 2.679(3) \AA$ & $1 \times 2.666(2)$ \\
\hline $\mathrm{Pb}-\mathrm{O}(1)$ & $1 \times 2.77(18) \AA$ & $1 \times 3.153(2) \AA$ & $1 \times 3.155(2)$ \\
\hline $\mathrm{Pb}-\mathrm{O}(2)$ & $1 \times 2.88(17) \AA$ & $1 \times 2.499(7) \AA$ & $1 \times 2.487(7)$ \\
\hline $\mathrm{Pb}-\mathrm{O}(2)$ & $1 \times 2.73(18) \AA$ & $1 \times 2.820(8) \AA$ & $1 \times 2.803(8)$ \\
\hline $\mathrm{Pb}-\mathrm{O}(2)$ & $1 \times 2.76(18) \AA$ & $1 \times 2.724(7) \AA$ & $1 \times 2.725(7)$ \\
\hline $\mathrm{Pb}-\mathrm{O}(2)$ & $1 \times 2.83(17) \AA$ & $1 \times 3.163(7) \AA$ & $1 \times 3.183(7)$ \\
\hline $\mathrm{Pb}-\mathrm{O}(3)$ & $1 \times 2.455(18) \AA$ & $1 \times 2.491(7) \AA$ & $1 \times 2.495(7)$ \\
\hline $\mathrm{Pb}-\mathrm{O}(3)$ & $1 \times 3.296(11) \AA$ & $1 \times 2.812(8) \AA$ & $1 \times 2.819(8)$ \\
\hline $\mathrm{Pb}-\mathrm{O}(3)$ & $1 \times 2.415(11) \AA$ & $1 \times 2.732(7) \AA$ & $1 \times 2.715(7)$ \\
\hline $\mathrm{Pb}-\mathrm{O}(3)$ & $1 \times 3.223(18) \AA$ & $1 \times 3.176(7) \AA$ & $1 \times 3.179(7)$ \\
\hline $\mathrm{Ni}-\mathrm{O}(1)$ & $2 \times 2.01(5) \AA$ & $2 \times 2.091(6) \AA$ & $2 \times 2.090(6)$ \\
\hline $\mathrm{Ni}-\mathrm{O}(2)$ & $2 \times 1.98(5) \AA$ & $2 \times 2.079(5) \AA$ & $2 \times 2.067(5)$ \\
\hline $\mathrm{Ni}-\mathrm{O}(3)$ & $2 \times 2.275(13) \AA$ & $2 \times 2.084(4) \AA$ & $2 \times 2.074(5)$ \\
\hline $\mathrm{Os}-\mathrm{O}(1)$ & $2 \times 1.95(5) \AA$ & $2 \times 1.910(6) \AA$ & $2 \times 1.914(5)$ \\
\hline $\mathrm{Os}-\mathrm{O}(2)$ & $2 \times 1.99(5) \AA$ & $2 \times 1.935(5) \AA$ & $2 \times 1.941(5)$ \\
\hline $\mathrm{Os}-\mathrm{O}(3)$ & $2 \times 1.841(13) \AA$ & $2 \times 1.933(4) \AA$ & $2 \times 1.940(5)$ \\
\hline $\mathrm{Os}-\mathrm{O}(1)-\mathrm{Ni}$ & $179.5(12)^{\circ}$ & $159.20(9)^{\circ}$ & $158.79(9)$ \\
\hline $\mathrm{Os}-\mathrm{O}(2)-\mathrm{Ni}$ & $175.4(12)^{\circ}$ & $161.3(4)^{\circ}$ & $161.0(4)$ \\
\hline $\mathrm{Os}-\mathrm{O}(3)-\mathrm{Ni}$ & $147.1(4)^{\circ}$ & $160.5(4)^{\circ}$ & $160.0(4)$ \\
\hline $\mathrm{BVS}_{(\mathrm{Ni})}$ & +2.08 & +1.98 & +2.03 \\
\hline $\mathrm{BVS}_{(\mathrm{Os})}$ & +5.98 & +5.91 & +5.82 \\
\hline
\end{tabular}


(a) 15

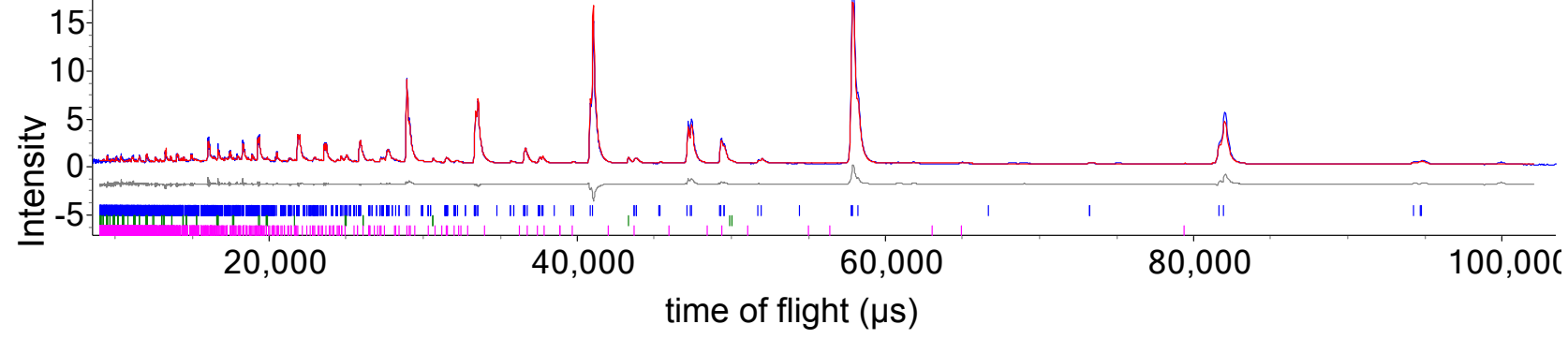

(b) 15

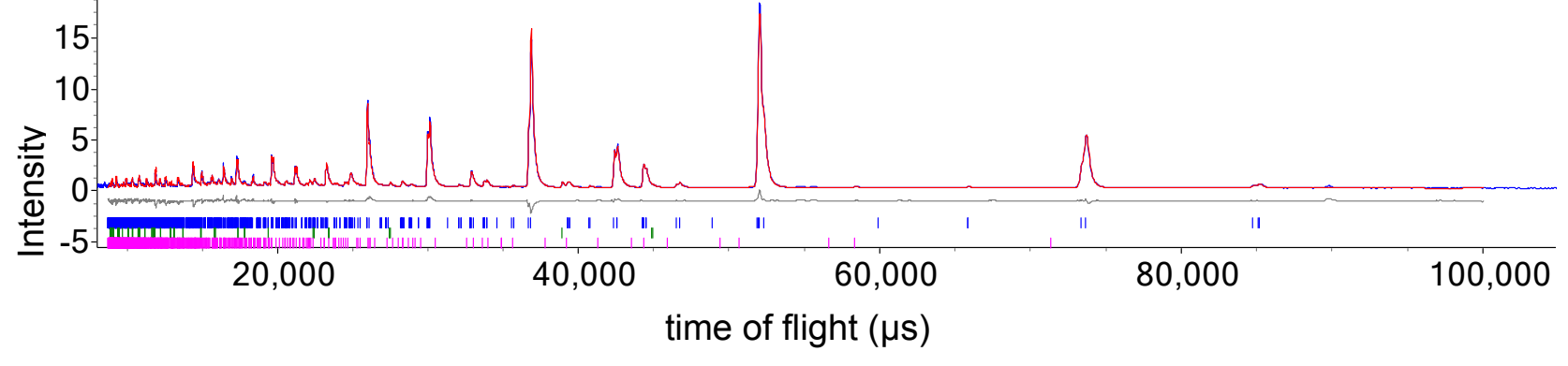

(c)

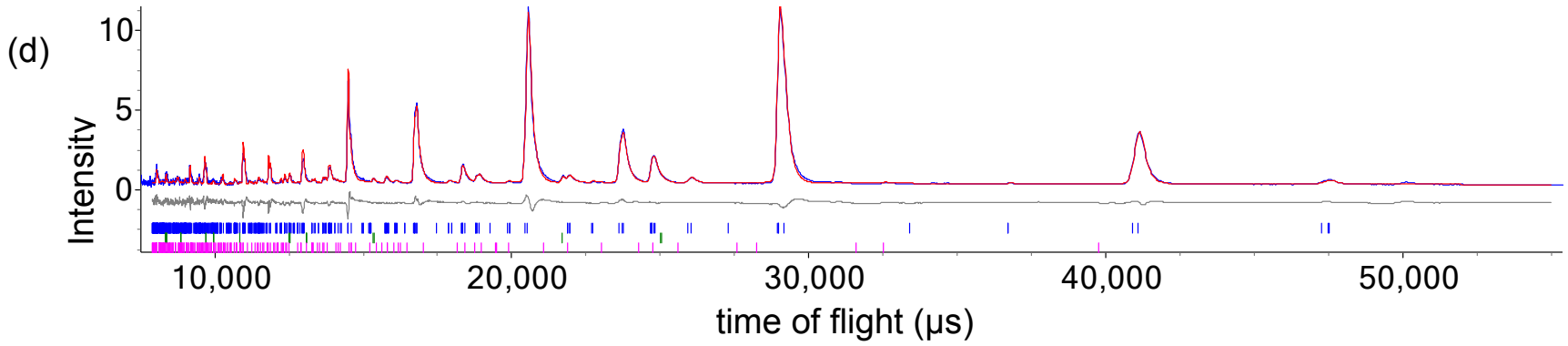

Figure S2a Refinement profiles (time of flight) for $\mathrm{Pb}_{2} \mathrm{NiOsO}_{6}$ using $98 \mathrm{~K}$ NPD data in space group $P 2_{1} / n$ showing (a) higher resolution ( $153^{\circ}$ bank) data, (b) $122^{\circ}$ bank data, (c) $90^{\circ}$ bank data and (d) $58^{\circ}$ bank data. Observed, calculated and difference profiles are shown in blue, red and grey, respectively; upper (blue), middle (green) and lower (pink) ticks show reflection positions for the main phase $\mathrm{Pb}_{2} \mathrm{NiOsO}_{6}(98.86(4) \%$ by weight) and impurity phases $\mathrm{NiO}(0.61(2) \%$ by weight) and $\mathrm{PbO}_{2}(0.53(3) \%$ by weight), respectively. 

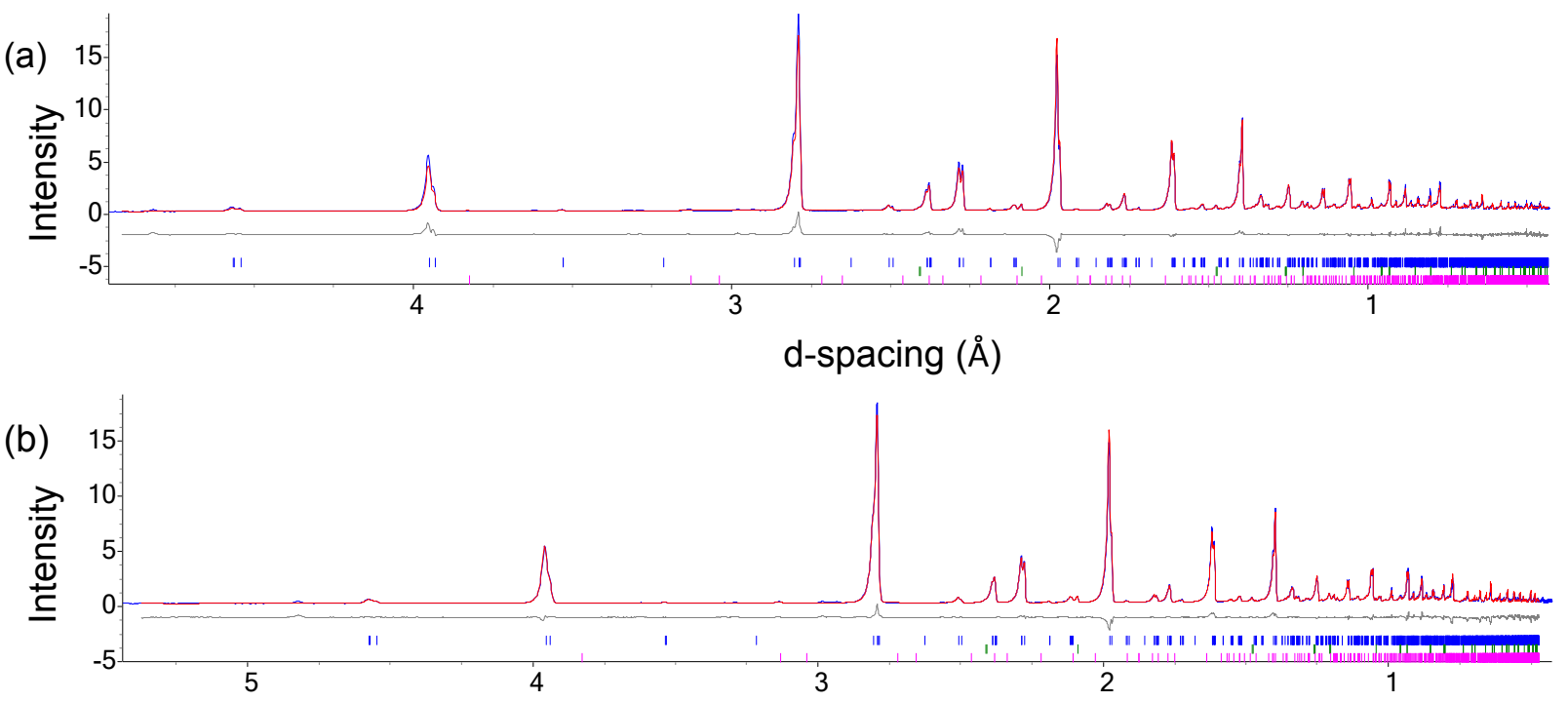

d-spacing ( $\AA$ )

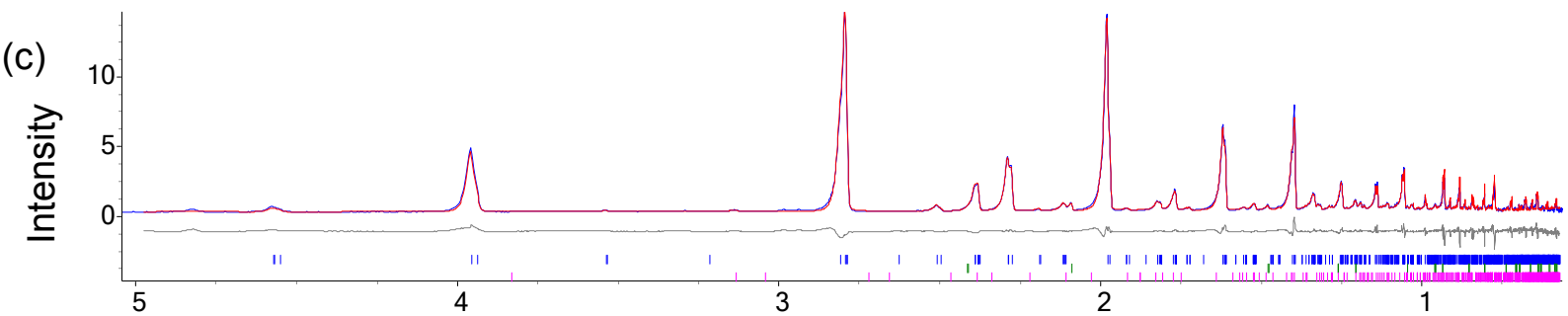

d-spacing ( $(\AA)$

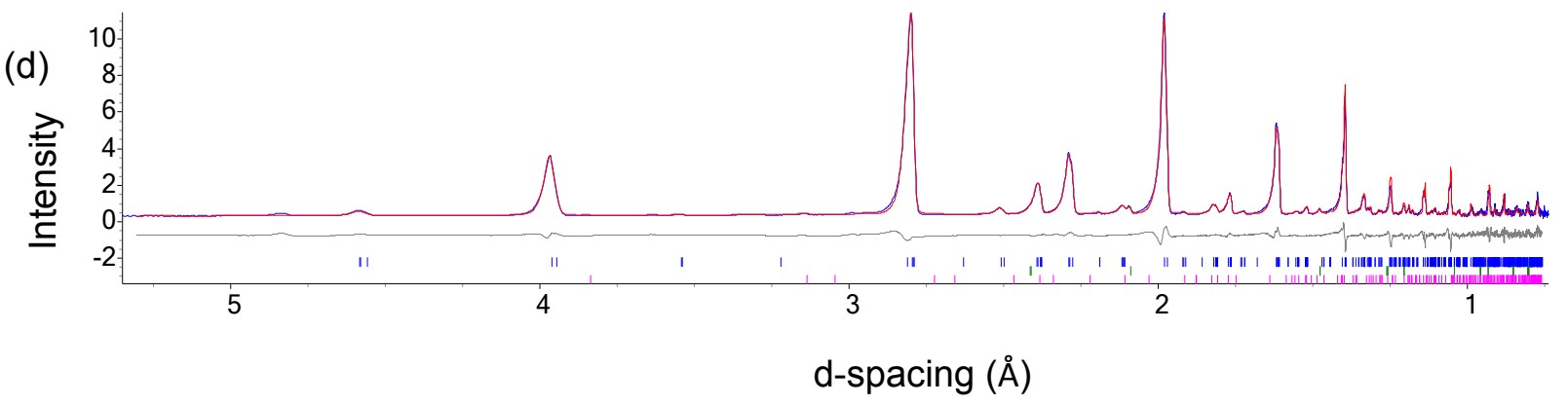

Figure S2b Refinement profiles (d-spacing) for $\mathrm{Pb}_{2} \mathrm{NiOsO}_{6}$ using $98 \mathrm{~K}$ NPD data in space group $P 2_{1} / n$ showing (a) higher resolution ( $153^{\circ}$ bank) data, (b) $122^{\circ}$ bank data, (c) $90^{\circ}$ bank data and (d) $58^{\circ}$ bank data. Observed, calculated and difference profiles are shown in blue, red and grey, respectively; upper (blue), middle (green) and lower (pink) ticks show reflection positions for the main phase $\mathrm{Pb}_{2} \mathrm{NiOsO}_{6}(98.86(4) \%$ by weight) and impurity phases $\mathrm{NiO}(0.61(2) \%$ by weight) and $\mathrm{PbO}_{2}(0.53(3) \%$ by weight), respectively. 
Table S3 Nuclear refinement details from Rietveld refinement of $\mathrm{Pb}_{2} \mathrm{NiOsO}_{6}$ using $98 \mathrm{~K}$ (upper values) and $1.5 \mathrm{~K}$ (lower values) NPD data in space group $P 2_{1} / n$. At $98 \mathrm{~K}$, cell parameters are $a=$ 5.6134(1) $\AA, b=5.5717(1) \AA, c=7.8757(2) \AA, \beta=89.956(4)^{\circ} ; R_{w p}=6.07 \%, R_{p}=6.70 \%$. At $1.5 \mathrm{~K}$, cell parameters are $a=5.60954(5) \AA, b=5.57031(4) \AA, c=7.87140(5) \AA, \beta=89.958(3)^{\circ}$; $R_{\mathrm{wp}}=5.90 \%, R_{\mathrm{p}}=6.34 \%$. The values obtained from $1.5 \mathrm{~K} \mathrm{NPD}$ data are given on the second row for each atom.

\begin{tabular}{|c|c|c|c|c|c|c|c|}
\hline Atom & site & $x$ & $y$ & $z$ & $\begin{array}{l}\text { Factional } \\
\text { occupancy }\end{array}$ & $\begin{array}{l}U_{\text {iso }} \times \\
100\left(\AA^{2}\right)\end{array}$ & $\begin{array}{l}\text { Magnetic } \\
\text { moment } \\
\left(\mu_{\mathrm{B}}\right)\end{array}$ \\
\hline \multirow[t]{2}{*}{$\mathrm{Pb}$} & \multirow[t]{2}{*}{$4 e$} & $0.0027(2)$ & $0.5142(2)$ & $0.2495(7)$ & $0.970(1) \dagger$ & $0.90(2)$ & - \\
\hline & & $0.0030(2)$ & $0.5161(2)$ & $0.2483(8)$ & $0.970(1) \dagger$ & $0.50(1)$ & - \\
\hline \multirow[t]{2}{*}{$\mathrm{Ni}$} & \multirow[t]{2}{*}{$2 a$} & 0 & 0 & 0 & $1.00(6) \dagger$ & $1.07(2)^{*}$ & - \\
\hline & & 0 & 0 & 0 & $1.00(6) \dagger$ & $0.74(1)^{*}$ & $1.90(1)$ \\
\hline \multirow[t]{2}{*}{ Os } & \multirow[t]{2}{*}{$2 b$} & 0 & 0 & 0.5 & $1.00(6) \dagger$ & $1.07(2)^{*}$ & - \\
\hline & & 0 & 0 & 0.5 & $1.00(6) \dagger$ & $0.74(1)^{*}$ & $0.378(2)$ \\
\hline \multirow[t]{2}{*}{$\mathrm{O}(1)$} & \multirow[t]{2}{*}{$4 e$} & $-0.0635(2)$ & $-0.0100(4)$ & $0.2615(7)$ & $0.988(3) \dagger$ & $0.91(7)$ & - \\
\hline & & $-0.0647(2)$ & $-0.0104(3)$ & $0.2614(7)$ & $0.988(3) \dagger$ & $0.55(6)$ & - \\
\hline \multirow[t]{2}{*}{$\mathrm{O}(2)$} & \multirow[t]{2}{*}{$4 e$} & $0.2342(7)$ & $0.2854(8)$ & $0.0323(9)$ & $0.948(7) \dagger$ & $0.8(1)$ & - \\
\hline & & $0.2315(8)$ & $0.2850(8)$ & $0.0328(9)$ & $0.948(7) \dagger$ & $0.5(1)$ & - \\
\hline \multirow[t]{2}{*}{$\mathrm{O}(3)$} & \multirow[t]{2}{*}{$4 e$} & $0.2874(6)$ & $0.7680(8)$ & $0.0330(8)$ & $1.013(7) \dagger$ & $1.5(1)$ & - \\
\hline & & $0.2863(7)$ & $0.7694(8)$ & $0.0338(9)$ & $1.013(7) \dagger$ & 1.1(1) & - \\
\hline
\end{tabular}

$\dagger$ occupancies fixed at values from refinement with a single global $U_{\text {iso }}$ (see text)

* constrained to be identical

(a)

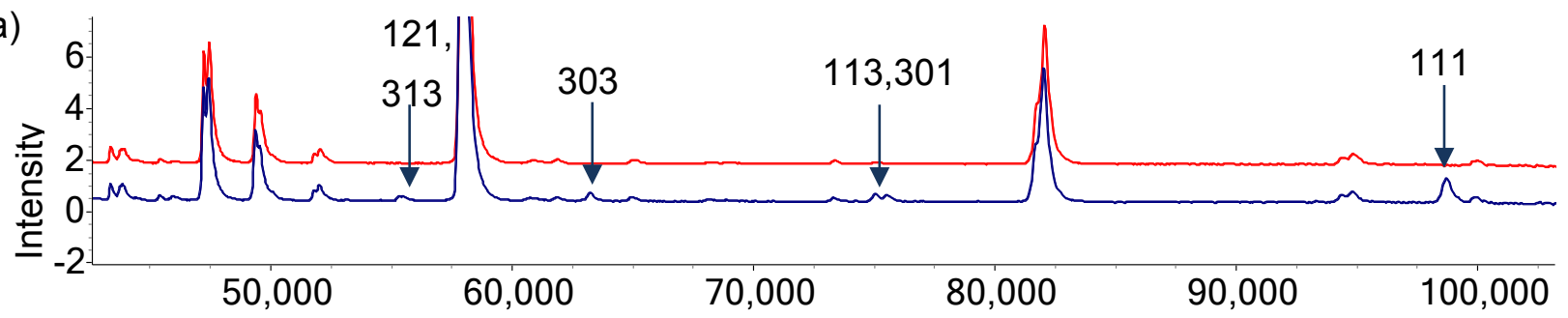

(b)

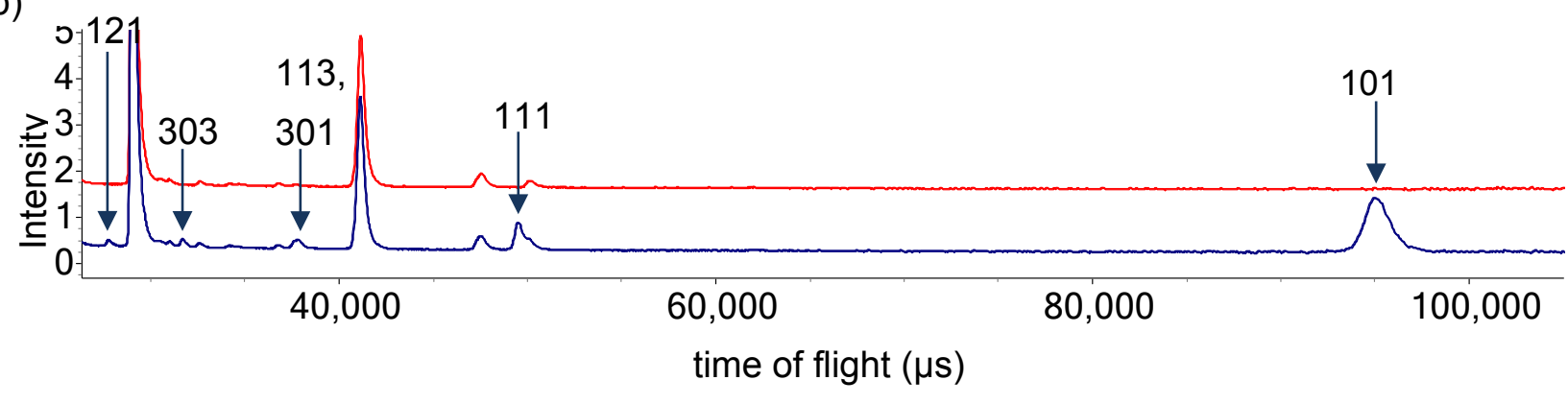

Figure S3 Raw NPD data for $\mathrm{Pb}_{2} \mathrm{NiOsO}_{6}$ collected at $98 \mathrm{~K}$ (red) and $2 \mathrm{~K}$ (blue) with additional magnetic reflections indexed by a magnetic unit cell with dimensions $2 a_{n} \times b_{n} \times 2 c_{n}$ (subscript $n$ refers to the nuclear unit cell) showing (a) higher resolution ( $153^{\circ}$ bank) data and (b) $58^{\circ}$ bank data. 

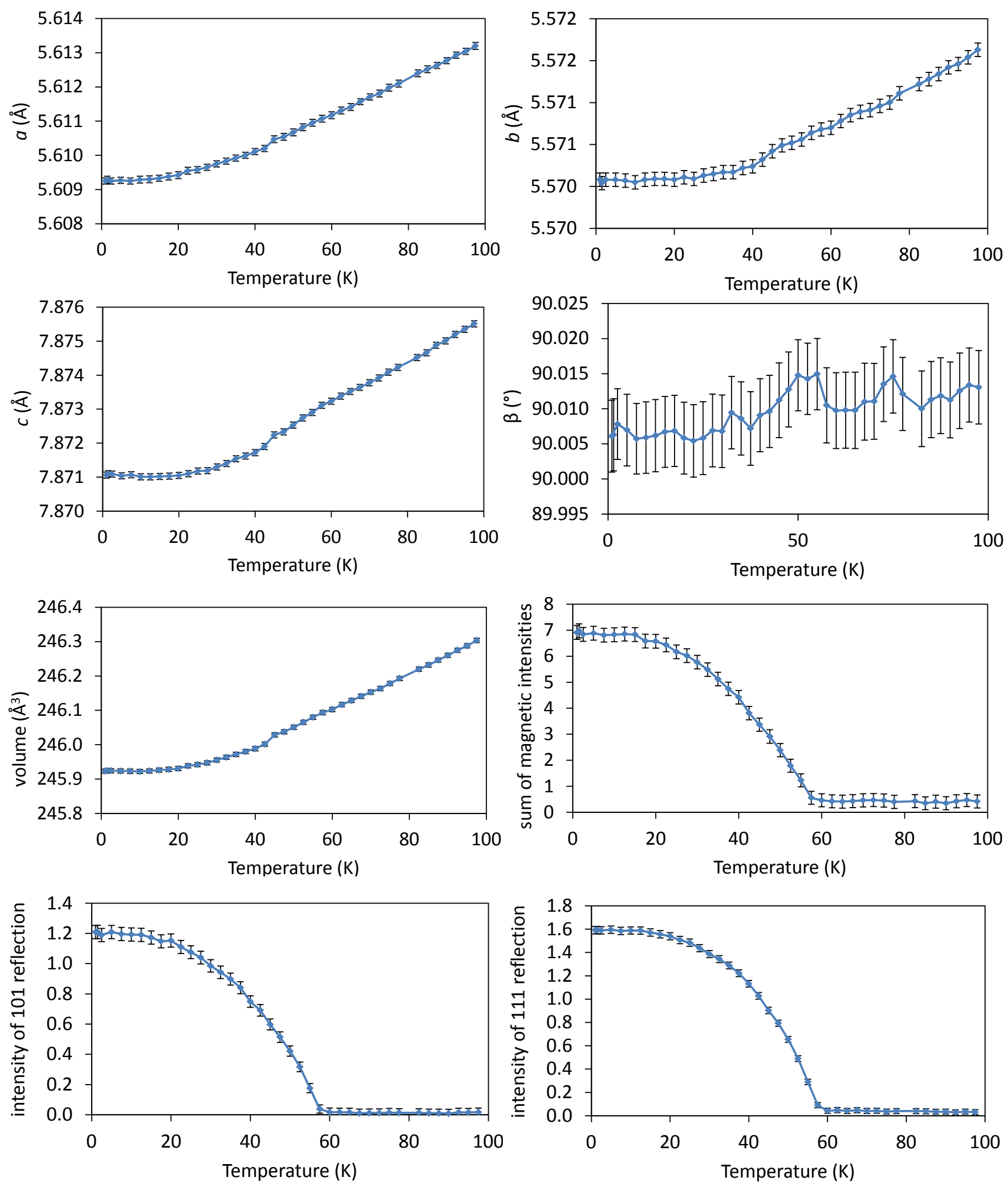

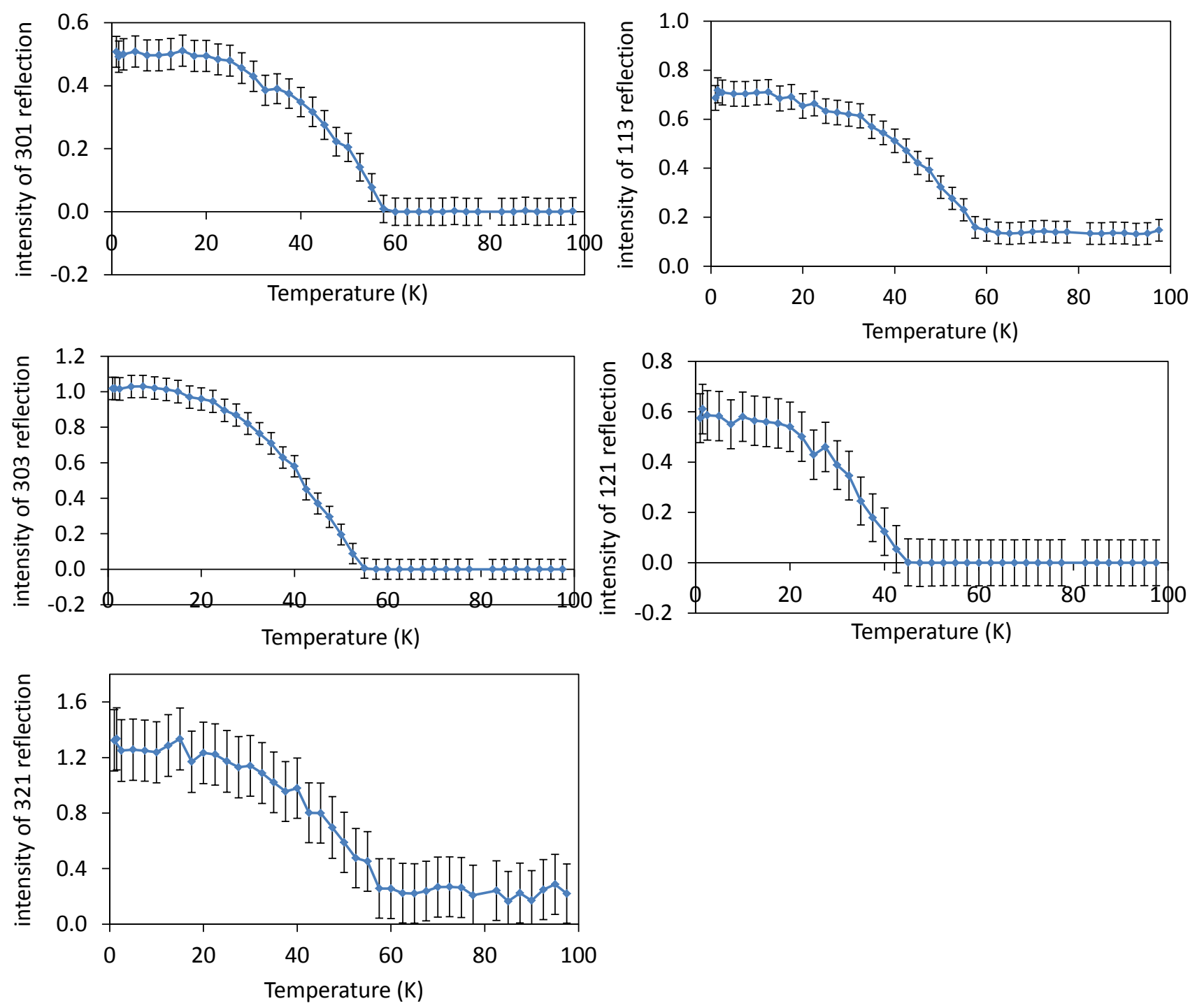

Figure S4 Evolution of magnetic peak intensity with temperature for $\mathrm{Pb}_{2} \mathrm{NiOsO}_{6}$ from sequential refinements using $58^{\circ}$ bank NPD data: the nuclear phase was fitted with a Rietveld phase with unit cell parameters determined from refinement using high resolution $\left(153^{\circ}\right.$ bank) data and the magnetic reflections were fitted with a Pawley phase with unit cell dimensions dimensions $2 a_{n}$ $\times b_{n} \times 2 c_{n}$ (subscript $n$ refers to the nuclear unit cell) and intensities of key reflections output. 


\section{Comparison of possible magnetic models and magnetic refinement details:}

In addition to the $\mathrm{Os} \mathrm{mY}_{2}{ }^{-}$magnetic order, allowing $\mathrm{mY}_{1}{ }^{+}$moments on $\mathrm{Ni}$ sites and $\mathrm{\Gamma}_{2}{ }^{-}$displacements lowers $R_{\text {wp }}$ from $6.42 \%$ to $5.99 \%$, while allowing $\mathrm{mY}_{2}{ }^{+}$moments on $\mathrm{Ni}$ sites and $\Gamma_{1}{ }^{-}$displacements lowers $R_{w p}$ to $5.97 \%$. Attempts were made to follow the magnitude of the $\Gamma_{1}{ }^{-}$and $\Gamma_{2}$ - displacements in sequential refinements to study their temperature dependence but any such displacements must be subtle and neither varied continuously with temperature.

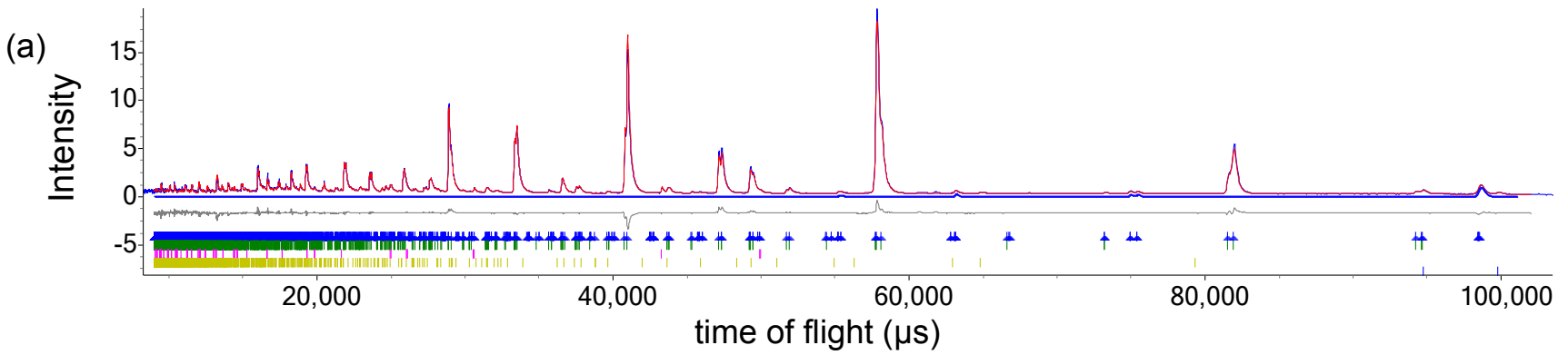

(b)

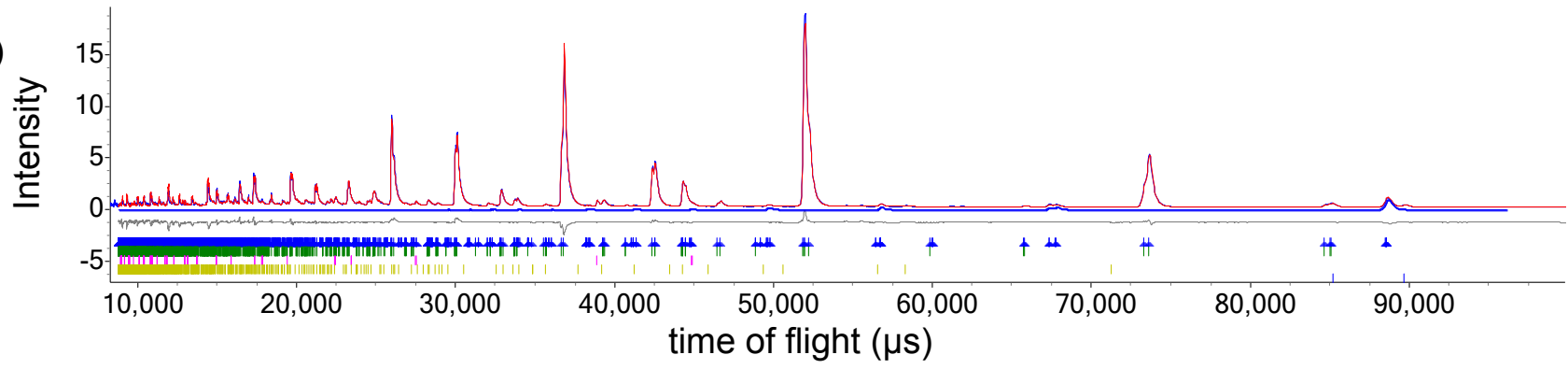

(c)

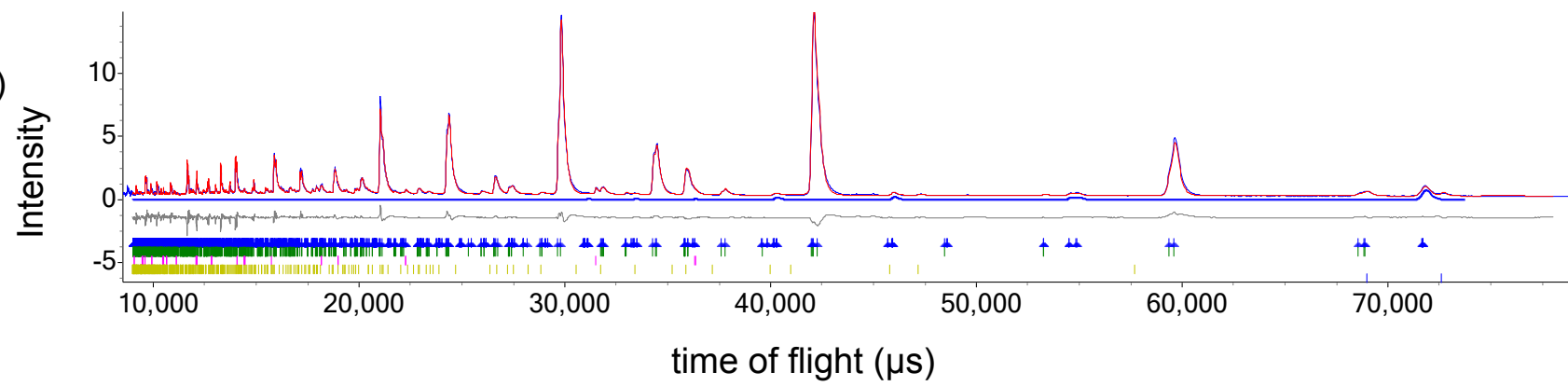

(d)

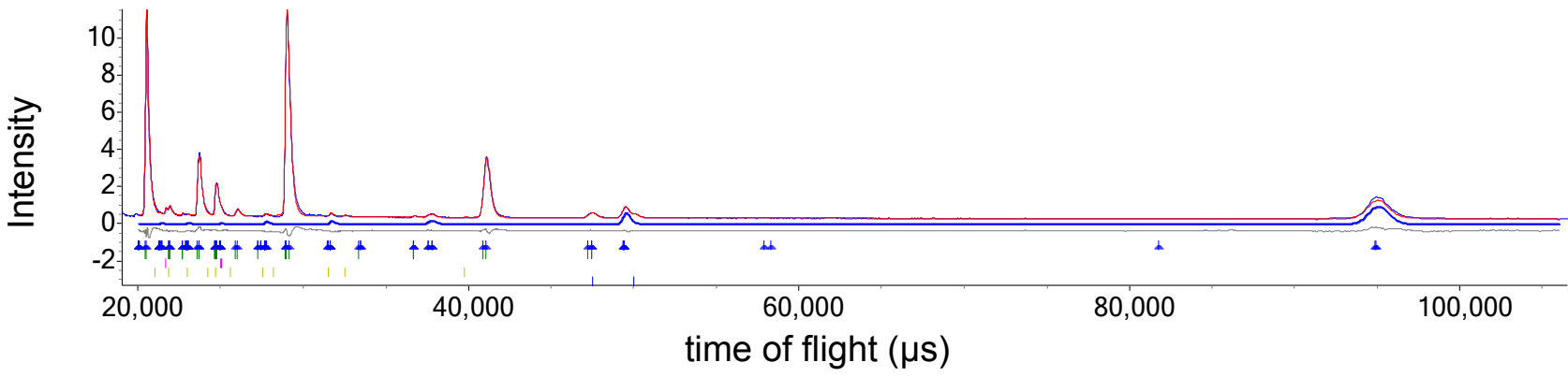

Figure S5a Refinement profiles (in time of flight) for $\mathrm{Pb}_{2} \mathrm{NiOsO}_{6}$ using $1.5 \mathrm{~K} \mathrm{NPD}$ data in space group $P 2_{1} / n$ showing (a) higher resolution ( $153^{\circ}$ bank) data, (b) $122^{\circ}$ bank data, (c) $90^{\circ}$ bank data and (d) $58^{\circ}$ bank data. Observed, calculated and difference profiles are shown in blue, red and grey, respectively and magnetic scattering is highlighted in blue. Top (blue), upper-middle (green), middle (pink), lower (yellow) and bottom (blue) ticks show reflection positions for the magnetic phase, the main phase $\mathrm{Pb}_{2} \mathrm{NiOsO}_{6}$ ( $\sim 99 \%$ by weight), impurity phases $\mathrm{NiO}(\sim 0.6 \%$ by weight), and $\mathrm{PbO}_{2}(\sim 0.5 \%$ by weight $)$ and a Pawley phase to fit the two magnetic peaks of antiferromagnetic $\mathrm{NiO}$, respectively. 

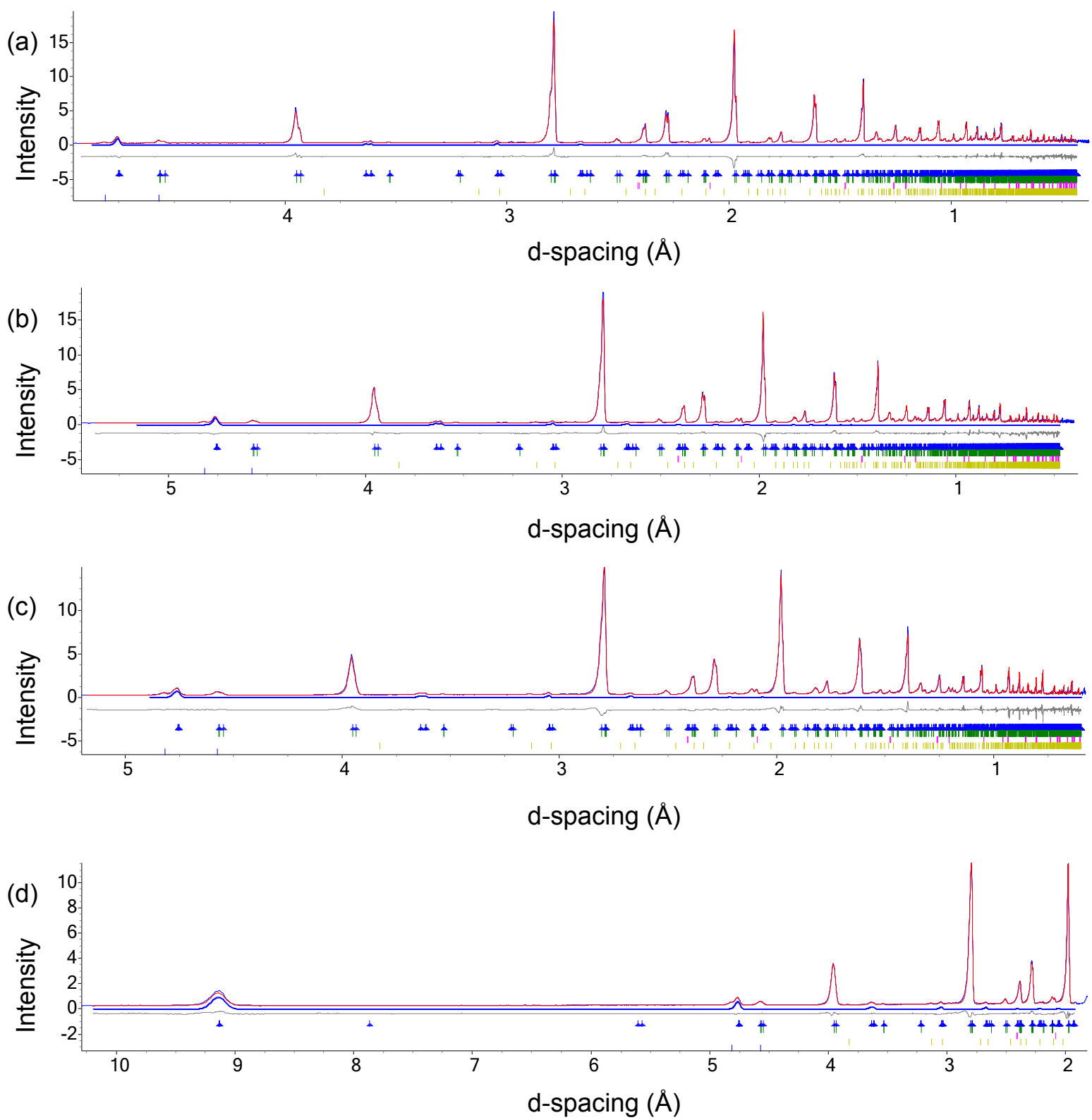

Figure S5b Refinement profiles (in d-spacing) for $\mathrm{Pb}_{2} \mathrm{NiOsO}_{6}$ using $1.5 \mathrm{~K}$ NPD data in space group $P 2_{1} / n$ showing (a) higher resolution ( $153^{\circ}$ bank) data, (b) $122^{\circ}$ bank data, (c) $90^{\circ}$ bank data and (d) $58^{\circ}$ bank data. Observed, calculated and difference profiles are shown in blue, red and grey, respectively and magnetic scattering is highlighted in blue. Top (blue), upper-middle (green), middle (pink), lower (yellow) and bottom (blue) ticks show reflection positions for the magnetic phase, the main phase $\mathrm{Pb}_{2} \mathrm{NiOsO}_{6}$ ( $\sim 99 \%$ by weight), impurity phases $\mathrm{NiO}(\sim 0.6 \%$ by weight), and $\mathrm{PbO}_{2}(\sim 0.5 \%$ by weight $)$ and a Pawley phase to fit the two magnetic peaks of antiferromagnetic $\mathrm{NiO}$, respectively. 

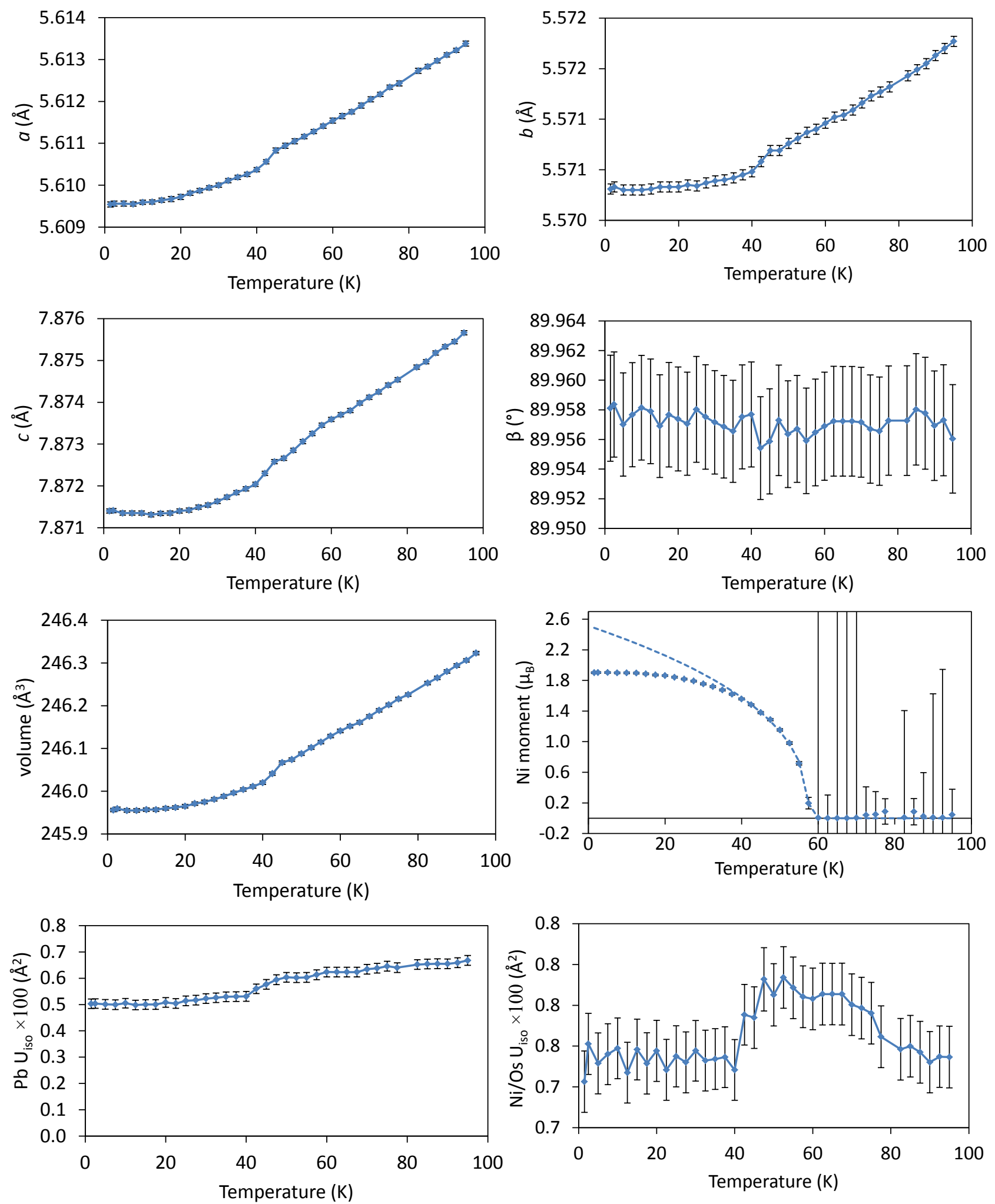

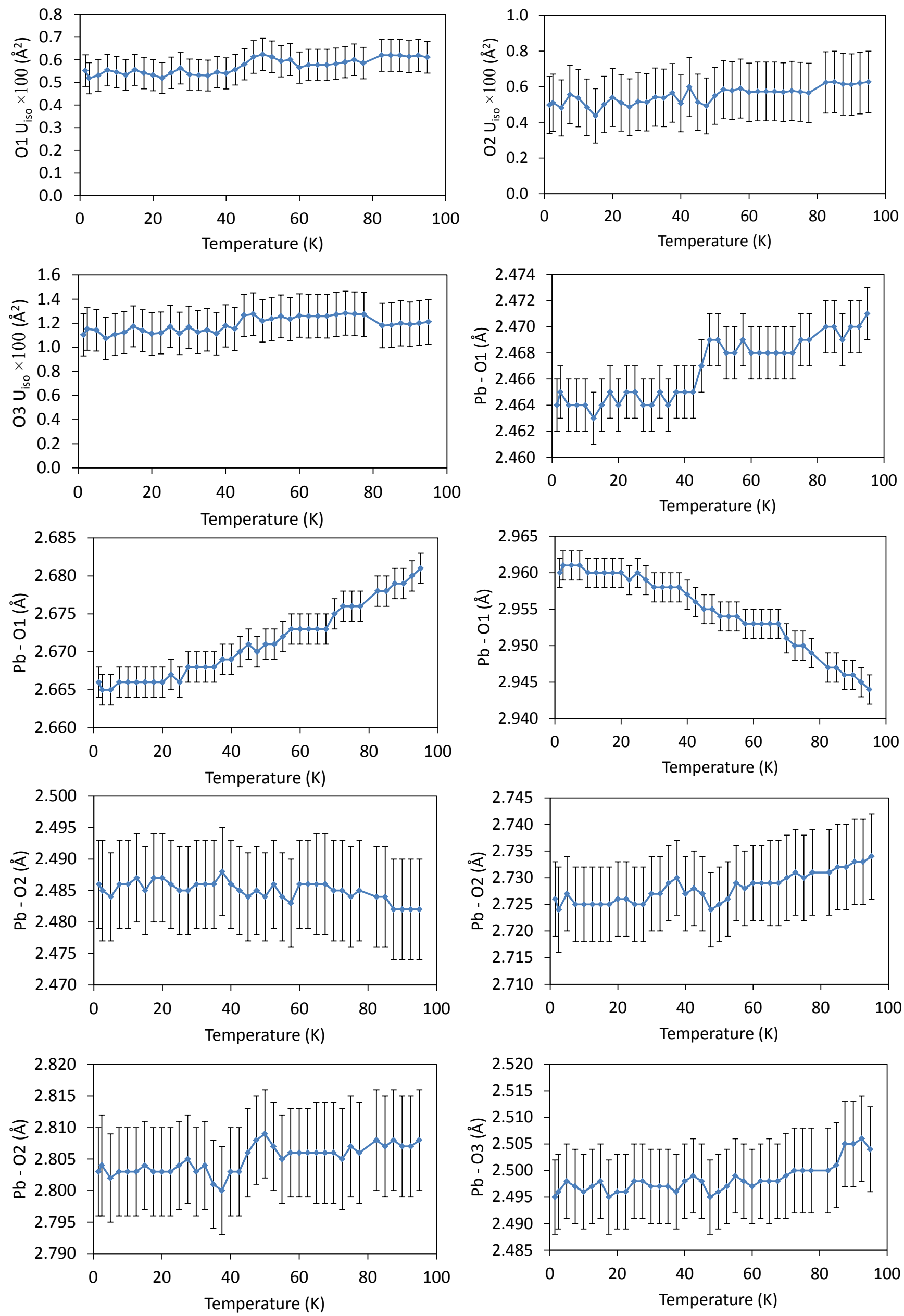

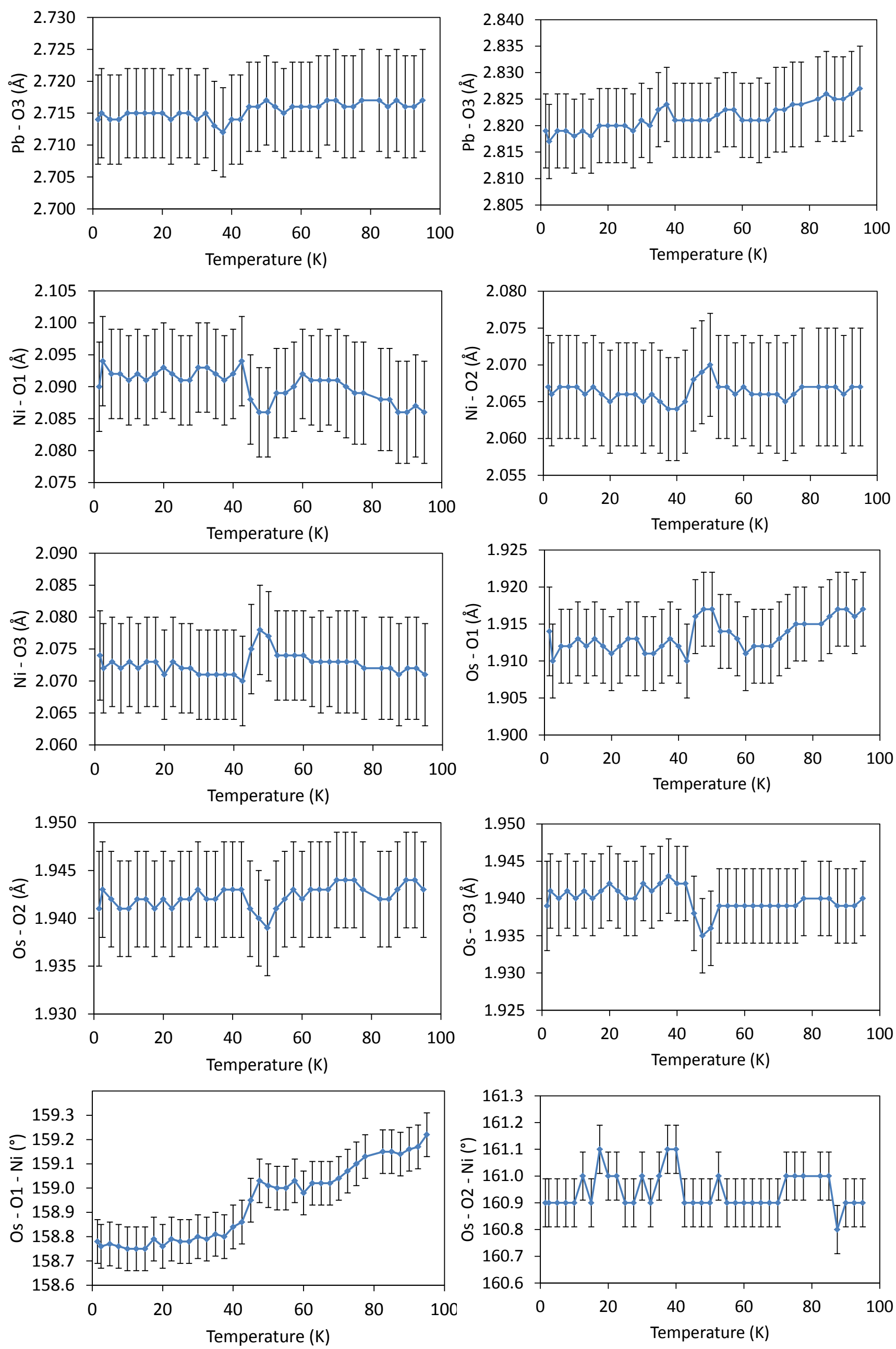


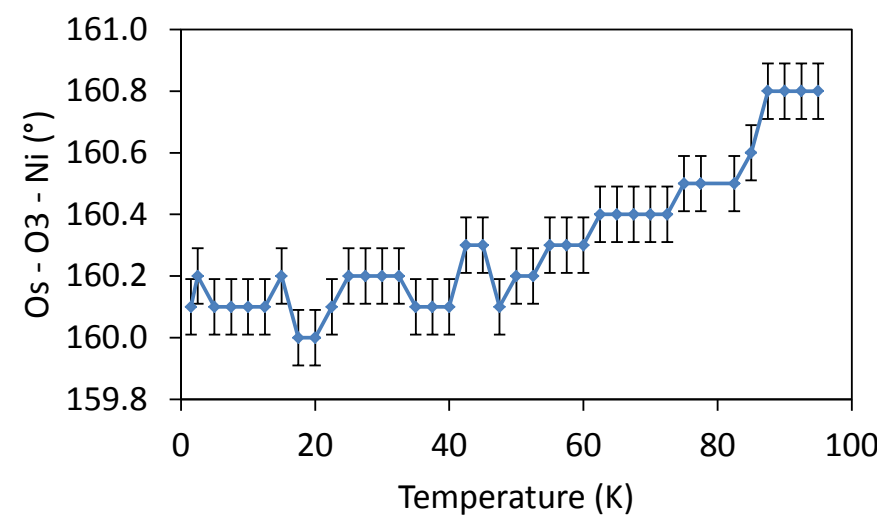

Figure 6 Evolution of nuclear and magnetic structures with temperature for $\mathrm{Pb}_{2} \mathrm{NiOsO}_{6}$ from sequential refinements using NPD data from higher resolution $\left(153^{\circ}\right)$ bank, $122^{\circ}$ bank, $90^{\circ}$ bank data and $58^{\circ}$ bank data. The nuclear phase was fitted with a Rietveld phase, as was the magnetic-only phase with unit cell parameters constrained to be related to the nuclear unit cell.

The unit cell volume contracts a little more rapidly on cooling below $\sim 40 \mathrm{~K}$ and this coincides with a change in the slope of some bond lengths and angles $(\mathrm{aPb}-\mathrm{O} 1$ and the $\mathrm{Os}-\mathrm{O} 1-\mathrm{Ni}$ bond angle) as the $\mathrm{O}(1)$ site moves in the negative $x$ direction. We note that the weakest magnetic reflection, 121 , appears to increase in intensity only below $\sim 40 \mathrm{~K}$ and there may be a slight change in magnetic structure (and a concomitant change in the crystal structure) at this temperature. Possible changes in the magnetic structure include ordering of a second magnetic sublattice e.g. if $\mathrm{Ni}^{2+}$ moments ordered at $T_{\mathrm{N}}$ and $\mathrm{Os}^{6+}$ moments ordered below $\sim 40 \mathrm{~K}$, or if some reorientation of moments occurred below $\sim 40 \mathrm{~K}$. Mode inclusion analysis ${ }^{1,2}$ using $47.5 \mathrm{~K}$ data gave qualitatively similar results to that carried out at low temperature (indicating the same magnetic structure). Likewise, refinements using $47.5 \mathrm{~K}$ data allowing the magnetic moment direction to refine freely, and constraining the moments to lie along the $P 2_{1} / n[001]$ direction gave very similar fits $\left(\mathrm{R}_{\mathrm{wp}} \mathrm{S}\right.$ of $5.938 \%$ and $5.936 \%$ for the constrained and free models, respectively) and the unconstrained model resulted in moments lying very close to the $P 2_{1} / n$ [001]. Due to the low intensity of the 121 reflection, it's hard to determine whether it is present for $40 \mathrm{~K} \leq T \leq T_{\mathrm{N}}$. Given the high correlation in the refinement between moments on the two cation sites, it may not be possible to resolve this question from powder diffraction data, and given the magnetic susceptibility and heat capacity data, we must assume a single magnetic ordering transition at $T_{\mathrm{N}}$. 


\section{Further details regarding DFT calculations:}

The magnetic ground state of $\mathrm{Pb}_{2} \mathrm{NiOsO}_{6}$ was studied by calculating the total energy for each of the different magnetic states, namely FM, AFM-1, and AFM-2 (see Figure S7). Both Ni and Os moments order in AFM-1, which leads to inversion symmetry break. On the other hand, only Ni moments order in AFM-2 and inversion symmetry is preserved. From the total energy calculations (see Table 2), AFM-1 is found to have the lowest energy, with an energy difference of $0.102 \mathrm{eV} /$ formula unit from that of AFM-2, thereby suggesting that DFT calculations favor the noncentrosymmetric AFM model with AFM order on both $\mathrm{Ni}$ and Os sublattices.

FM

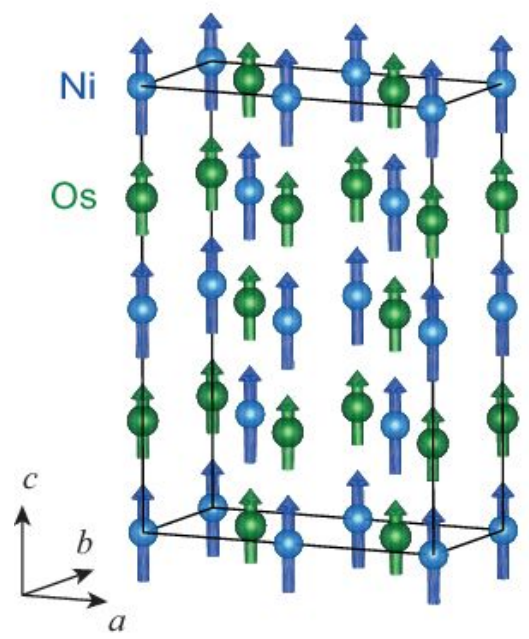

AFM-1

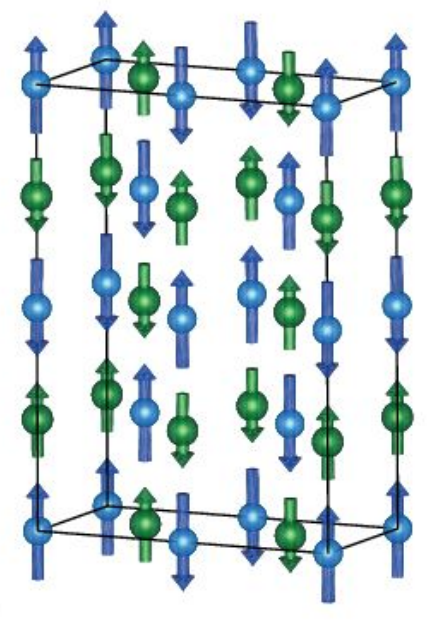

AFM-2

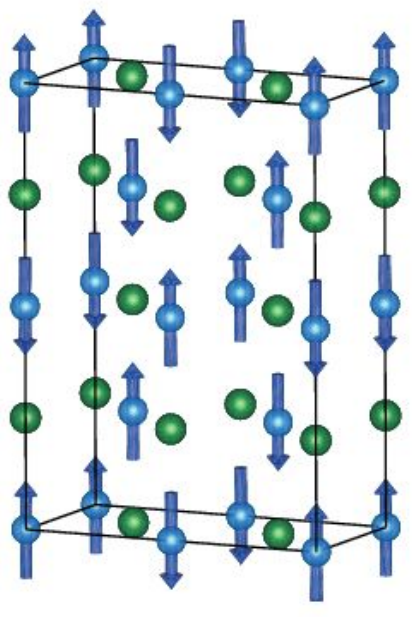

Figure S7 Possible spin arrangement considered in the theoretical calculation for $\mathrm{Pb}_{2} \mathrm{NiOsO}_{6}$; ferromagnetic (FM), antiferromagnetic AFM-1 and AFM-2 configurations. Ni and Os atoms are only shown for clarity.

Table S4 The total energy calculated for $\mathrm{Pb}_{2} \mathrm{NiOsO}_{6}$ with different magnetic states

Magnetic phase

Total energy (eV/formula unit)

AFM-1

AFM-2

FM
0

$+0.102$

$+1.880$ 
DFT calculations were carried out on the four magnetic models (models $1-4$, see main text) to investigate their relative energies. Details of these models including basis vectors and origin shifts relative to the nuclear $P 2{ }_{1} / n$ structure are given below. ${ }^{3}$

\section{Model 1:}

$\mathrm{Ni}: \mathrm{mY}_{2}^{+}$

Os: $\mathrm{mY}_{2}^{-}$

Polar distortion: $\Gamma_{1}^{-}$

Magnetic symmetry: $P_{a} 2_{1}$

Basis vectors: $(200)(010)(-101)$

Origin shift: $(-1 / 401 / 4)$

\section{Model 2:}

$\mathrm{Ni}: \mathrm{mY}_{1}^{+}$

Os: $\mathrm{mY}_{2}^{-}$

Polar distortion: $\Gamma_{2}^{-}$

Magnetic symmetry: $P_{a} C$

Basis vectors: (-200) (0-10) (101)

Origin shift: $\left(\begin{array}{lll}0 & 1 / 4 & 0\end{array}\right)$

\section{Model 3:}

$\mathrm{Ni}: \mathrm{mY}_{1}^{+}$

Os: $\mathrm{mY}^{-}$

Polar distortion: $\Gamma_{1}{ }^{-}$

Magnetic symmetry: $P_{a} 2_{1}$

Basis vectors: (200) (010) (-101)

Origin shift: $(-1 / 40-1 / 4)$

\section{Model 4:}

$\mathrm{Ni}: \mathrm{mY}_{2}{ }^{+}$

Os: $\mathrm{mY}_{1}^{-}$

Polar distortion: $\Gamma_{2}{ }^{-}$

Magnetic symmetry: $P_{a} C$

Basis vectors: (200) (010) (-101)

Origin shift: $\left(\begin{array}{lll}0 & 1 / 4 & 0\end{array}\right)$

\section{References}

1. McCabe, E. E.; Stock, C.; Rodrigues, E. E.; Wills, A. S.; Taylor, J. W.; Evans, J. S. O., Weak spin interactions in Mott insulating $\mathrm{La}_{2} \mathrm{O}_{2} \mathrm{Fe}_{2} \mathrm{OSe}_{2}$. Phys. Rev. B 2014, 89, 100402(R).

2. Tuxworth, A. J.; McCabe, E. E.; Free, D. G.; Clark, S. J.; Evans, J. S. O., Structural Characterization and Physical Properties of the New Transition Metal Oxyselenide $\mathrm{La}_{2} \mathrm{O}_{2} \mathrm{ZnSe}_{2}$. Inorg. Chem. 2013, 52, 2078-2085.

3. Campbell, B. J.; Stokes, H. T.; Tanner, D. E.; Hatch, D. M., ISODISTORT. J. Appl. Cryst. 2006, 39, 607614. 